\title{
Kinetics of recombination processes
}

\author{
$\mathrm{H} \mathrm{Rauh \dagger}$ and A M Stoneham \\ Theoretical Physics Division, AERE Harwell, Oxfordshire, UK
}

Received 22 March 1984

\begin{abstract}
In order to solve nonlinear kinetic equations for recombination reactions when the production of participating species is driven externally at a time-dependent rate, we establish a framework based on the correspondence between these equations and a Schrödinger-type equation in which the product of the recombination constant with the production rate plays the role of the potential. The required solutions of the kinetic equations can therefore be obtained by solving the equivalent Schrödinger-type equation directly, or by identifying an appropriately chosen time-dependent function as a particular integral of this equation and deducing the production rate subsequently. Both methods are illustrated by various examples covering production rates with periodic as well as aperiodic time dependences. The approach is seen to reveal a variety of solutions which can be exploited to assist identification of the mechanisms operating and to aid extraction of rate constants in conjunction with the experiment.
\end{abstract}

\section{Introduction}

The formalisms developed widely to describe chemical kinetics (see, e.g., Kehlen et al 1974) have often a much broader validity. This is especially true of rate-equation approaches to sequences of reactions among species. Reactions of the sort

$$
\mathrm{AB} \rightleftharpoons \mathrm{A}+\mathrm{B}
$$

have features in common when A and B are atomic species reacting in the gas or liquid phase, when they are electronic carriers (electrons or holes, including polarons and solvated forms), or when they are solid-state defects, either intrinsic or extrinsic. Experiments to determine rate constants, and hence analyse the underlying mechanisms, usually contain two complications. The first arises from a driving term: one might apply an external field (irradiation by light, for instance) to dissociate the species $\mathrm{AB}$ at a rate $P(t)$ for times $t \geqslant 0$ :

$$
\mathrm{AB} \stackrel{P(t)}{\longrightarrow} \mathrm{A}+\mathrm{B} \text {. }
$$

The second complication is due to the fact that the differential equations describing how the populations involved vary with time may be nonlinear, as in the case of bimolecular recombination of mobile species $\mathrm{A}$ and $\mathrm{B}$ :

$$
\mathrm{A}+\mathrm{B} \stackrel{R}{\rightarrow} \mathrm{AB}
$$

+ Scholar of the Commission of the European Communities attached to Harwell April 1982 until March 1983; on leave from Fachbereich Physik der Universität Osnabrück, Postfach 4469, D-4500 Osnabrück, FR Germany. 
with an appropriate reaction constant $R$. This complication leads to three difficulties. Firstly, even if the reaction and the concentrations of species AB, A and B can all be monitored fairly precisely, it may still be hard to obtain accurate rate constants for mathematical and numerical reasons, e.g. near degeneracies of time constants. Secondly, the fit of the proposed rate equations may not yield unique values of rates, i.e. there may be several possible fits of comparable accuracy. Thirdly, since the experimental system may not be ideally defined, it may not be obvious how to vary the external field in order to optimise the approach.

In this paper therefore we look at general features of the kinetics of recombination processes typified by the reactions (1.1) when the production of species is driven at a rate $P(t)$. We shall show that there exist certain broad classes of solutions of the affiliated rate equations and that, in principle, these solutions can be exploited to improve analysis to give rate constants. Thus, if the production rate (which is under experimental control) is chosen with a suitable time dependence, one may optimise the response (e.g. photoconductivity (Mort and Pai 1976), or luminescence intensity (Curie 1963) versus time for reactions of electrons and holes) so as to aid extraction of rate constants. One significant point hereby concerns what constitutes a useful solution. Clearly analytic expressions for the instantaneous concentrations and recombination rate are always desirable. Under some circumstances more restricted results can be profitable. Thus, if for a periodic production rate the time scale of the fluctuations implied is small compared with the response time of the phenomenon being observed in the experiment, a description of the average concentrations and average recombination rate may be adequate. Hence, whilst emphasising the fully soluble cases, we shall quote results for averages too.

Chemical rate equations themselves involve certain approximations (see, e.g., Stoneham 1975) though few limitations concern the examples we discuss. The most critical approximation in the present analysis is probably the implied spatial homogeneity, so that a single production rate and a single recombination constant (or group of constants) apply. Clearly there are exceptions to the assumed uniformity. These might occur because the intensity of exciting radiation is attenuated as it penetrates deeper into the sample, or because nearby surfaces may offer extra recombination processes. For present purposes, we shall ignore this complication since, in some practical situations, a more restricted assumption of local homogeneity may suffice in any case.

\section{General formalism}

The basic rate equations considered here include three main types of terms which add to $\mathrm{d} C_{\alpha} / \mathrm{d} t$, the rate of change of concentration $C_{\alpha}$ of species $\alpha=\mathrm{A}, \mathrm{B}$. Firstly, there is the production term $P(t)$. For the reaction (1.2) the rates of production of species $A$ and $B$ are equal; clearly simple generalisations to more complex reactions exist. Secondly, there is a nonlinear recombination term $R C_{\mathrm{A}} C_{\mathrm{B}}$ due to reaction (1.3). However, we should not forget that species $\alpha$ can be generated by thermal ex citation, even in the absence of external interference. Therefore, if $\hat{C}_{\alpha}$ denotes the respective thermal equilibrium concentrations (assumed to be independent of $C_{\alpha}$ ), the full expression for the recombination rate becomes $L=R\left(C_{\mathrm{A}} C_{\mathrm{B}}-\hat{C}_{\mathrm{A}} \hat{C}_{\mathrm{B}}\right)$, which accounts for exact balance of the thermal production with recombination in equilibrium (cf Madelung 1981). We are hence led to the important class of coupled rate equations 
defined by

$\mathrm{d} C_{\alpha} / \mathrm{d} t=P(t)-R\left(C_{\mathrm{A}} C_{\mathrm{B}}-\hat{C}_{\mathrm{A}} \hat{C}_{\mathrm{B}}\right) \quad$ for $t \geqslant 0 \quad(\alpha=\mathrm{A}, \mathrm{B})$

together with initial values for $t=0$ :

$$
C_{\alpha}(0)=\hat{C}_{\alpha} \geqslant 0 \quad(\alpha=\mathrm{A}, \mathrm{B}) .
$$

Equations (2.1) subject to (2.2) obviously imply the conservation relation

$$
C_{\mathrm{A}}(t)-C_{\mathrm{B}}(t)=\hat{C}_{\mathrm{A}}-\hat{C}_{\mathrm{B}} \text {. }
$$

This allows us to represent them in the Riccati form

$$
\mathrm{d} C_{\alpha} / \mathrm{d} t=P(t)-R\left(C_{\alpha}^{2}+\hat{Q}_{\alpha} C_{\alpha}-\hat{C}_{\mathrm{A}} \hat{C}_{\mathrm{B}}\right) \quad(\alpha=\mathrm{A}, \mathrm{B})
$$

with the quantities

$$
\hat{Q}_{\mathrm{A}}=\hat{C}_{\mathrm{B}}-\hat{C}_{\mathrm{A}}, \quad \hat{Q}_{\mathrm{B}}=\hat{C}_{\mathrm{A}}-\hat{C}_{\mathrm{B}}
$$

introduced to abbreviate notation. When reactions (1.2) and (1.3) are the only ones involved, $\hat{Q}_{\alpha}=0$ for $\alpha=\mathrm{A}, \mathrm{B}$, and thus the linear term in (2.4) disappears. This applies, e.g., to carriers in perfectly pure intrinsic semiconductors, or ideally pure and perfect metals with displacement damage only. Yet, traps can alter the symmetry of $A$ and $\mathrm{B}$, so that $\hat{Q}_{\alpha} \neq 0$ for $\alpha=\mathrm{A}, \mathrm{B}$. Then, thirdly, (2.4) retains a linear term which generalises the basic equations whilst leaving an important class rigorously soluble.

We realise that the transformation

$$
C_{\alpha}(t)=R^{-1}(\mathrm{~d} / \mathrm{d} t) \log |Z(t)|-\frac{1}{2} \hat{Q}_{\alpha} \quad(\alpha=\mathrm{A}, \mathrm{B})
$$

relates the solution $C_{\alpha}(t)$ of the nonlinear first-order differential equations $(2.4)$ to an appropriate solution, $Z(t)$, of the linear second-order differential equation

$$
\mathrm{d}^{2} Z / \mathrm{d} t^{2}-\left[S^{2}+R P(t)\right] Z=0,
$$

in which

$$
S=R\left(\hat{C}_{\mathrm{A}}+\hat{C}_{\mathrm{B}}\right) / 2 .
$$

Although we are concerned with an initial value problem rather than an eigenvalue problem, we note formal parallels of (2.7) with the stationary Schrödinger equation for a particle in a one-dimensional potential, understanding that $t$ here represents the space variable. Thus, $Z(\mathrm{t})$ corresponds to the wavefunction, $-\mathrm{S}^{2}$ to the (negative) particle energy, and $R P(t)$ to the (non-negative) potential. The preceding steps reveal that, having established a value of $S$, one can exploit the whole class of (unstable) solutions of the Schrödinger-type equation (2.7) known for given forms of $P(t)$ to yield solutions $C_{\alpha}(t)$ of the original problem set up in equations (2.1) and (2.2). Conversely, starting from equation (2.7), one can obtain additional solutions of the rate equations under consideration by choosing $Z(t)$ appropriately, which then determines $P(t)$. In quantum mechanical terms, this corresponds to choosing a wavefunction and deducing the potential, i.e. the opposite of the usual method of solution. Both ways will be described and adopted in what follows.

\subsection{Solution with production rate given}

Here we choose a production rate $P(t)$ and derive concentrations $C_{\alpha}(t)$. When $P(t)$ is specified, equation (2.7) can be solved, in principle at least, to yield two linearly 
independent particular integrals, $Z_{1}(t)$ and $Z_{2}(t)$. Employing these in the transformation (2.6), and observing the conditions (2.2), leads to the following representation for the desired solution of equations (2.1):

$C_{\alpha}(t)=R^{-1}(\mathrm{~d} / \mathrm{d} t) \log |Z(t)|-\frac{1}{2} \hat{Q}_{\alpha} \quad$ for $t \geqslant 0 \quad(\alpha=\mathrm{A}, \mathrm{B})$

with $Z(t)$ given by

$$
Z(t)=k_{1} Z_{2}(t)-k_{2} Z_{1}(t)
$$

and constants $k_{1}$ and $k_{2}$ (apart from an unimportant non-zero factor) themselves given by

$$
k_{i}=Z_{i}(0)\left\{\left[(\mathrm{d} / \mathrm{d} t) \log \mid Z_{i}(t)\right]_{t=0}-S\right\} \quad(i=1,2) .
$$

From (2.9) the recombination rate can be calculated to yield

$$
L(t)=\frac{1}{R}\left\{[(\mathrm{~d} / \mathrm{d} t) \log |Z(t)|]^{2}-S^{2}\right\} \quad \text { for } t \geqslant 0
$$

using (2.5) and (2.8).

\subsection{Solution with production rate to be deduced}

We show next how, by choosing concentrations $C_{\alpha}(t)$ in effect, we can obtain the production rate $P(t)$. To do this, let us consider a function $Z_{1}(t)$ defined for times $t \geqslant 0$, and with continuous first derivative. This function is inserted into equation (2.7), and hence it constitutes another function, namely

$$
P(t)=R^{-1}\left[\left(Z_{1}(t)\right)^{-1} \mathrm{~d}^{2} Z_{1} / \mathrm{d} t^{2}-S^{2}\right] \quad \text { for } t \geqslant 0 .
$$

If, as a sufficient condition, the left-hand side of (2.13) turns out to be independent of $R$, bounded, and non-negative, thus satisfying the differential inequality

$$
\left(Z_{1}(t)\right)^{-1} \mathrm{~d}^{2} Z_{1} / \mathrm{d} t^{2} \geqslant S^{2} \quad \text { for } t \geqslant 0,
$$

then $P(t)$ from (2.13) may serve to model a production rate in equations (2.7) and (2.1). Since $P(t)$ is now known explicitly, we can identify $Z_{1}(t)$ as a first particular integral of the differential equation (2.7) and, by exploiting the Wronskian relationship, obtain a second linearly independent particular integral $Z_{2}(t)$ according to (Arfken 1970)

$$
Z_{2}(t) \propto Z_{1}(t) \int^{t} \frac{\mathrm{d} t^{\prime}}{Z_{1}^{2}\left(t^{\prime}\right)}
$$

In special cases we may be able to obtain $Z_{2}(t)$ more directly from $Z_{1}(t)$ by consideration of symmetry and linear independence, without making recourse to (2.15) explicitly. The subsequent calculation of the concentrations and of the recombination rate is straightforward, using (2.9) and (2.12).

This approach complements that of $\S 2.1$, as it requires satisfying the conditions associated with (2.13) to deduce $P(t)$, rather than solving the differential equation (2.7) for given $P(t)$. It is particularly useful for constructing simple solutions of the rate equations $(2.1)$. Thus, if $Z_{1}(t)$ is chosen such that

$$
\left[(\mathrm{d} / \mathrm{d} t) \log \left|Z_{1}(t)\right|\right]_{t=0}=S
$$


holds, this function already determines completely the concentrations from

$$
C_{\alpha}(t)=R^{-1}(\mathrm{~d} / \mathrm{d} t) \log \left|Z_{1}(t)\right|-\frac{1}{2} \hat{Q}_{\alpha} \quad(\alpha=A, B)
$$

and the recombination rate from

$$
L(t)=\frac{1}{R}\left\{\left[(\mathrm{~d} / \mathrm{d} t) \log \left|Z_{1}(t)\right|\right]^{2}-S^{2}\right\} .
$$

\subsection{Solution and averages for periodic production rate}

An important subclass is formed by solutions of the rate equations (2.1) with periodic time-dependence of $P(t)$. In this case (2.7) specifies a Hill equation (Meixner 1956). We may hence suppose that the relation

$$
P\left(t+\tau_{\mathrm{c}}\right)=P(t) \quad \text { for } t \geqslant 0
$$

applies, where $\tau_{\mathrm{c}}$ is the cycle length $\dagger$. Then, according to Floquet's theorem, there exist linearly independent particular integrals $Z_{1}(t)$ and $Z_{2}(t)$ of $(2.7)$ with the property

$$
Z_{1}\left(t+\tau_{\mathrm{c}}\right)=\mathrm{e}^{-\lambda \tau_{c}} Z_{1}(t), \quad Z_{2}\left(t+\tau_{\mathrm{c}}\right)=\mathrm{e}^{\lambda \tau_{c}} Z_{2}(t)
$$

The quantity $\lambda$ denotes the affiliated characteristic exponent which depends on $S^{2}$ and $R$ as well as on the parameters which characterise $P(t)$. Since $S^{2} \geqslant 0$ from (2.8) with (2.2) and since $P(t) \geqslant 0$ (excluding the trivial case of zero identity) from (2.13) with (2.14), $\lambda$ appears real and may be assumed positive without loss of generality. The statement $(2.20)$ is equivalent to the ansatz

$$
Z_{1}(t)=\mathrm{e}^{-\lambda t} u_{1}(t), \quad Z_{2}(t)=\mathrm{e}^{\lambda t} u_{2}(t)
$$

with periodic functions $u_{1}(t)$ and $u_{2}(t)$ satisfying

$$
u_{i}\left(t+\tau_{c}\right)=u_{i}(t) \quad \text { for } t \geqslant 0 \quad(i=1,2) .
$$

Again, by substituting the particular integrals (2.21) into (2.9) and (2.12), both the concentrations and the recombination rate are obtained. This reveals that the characteristic exponent plays the role of an inverse time constant for the establishment of steady state. Thus, if $t \gg 1 / \lambda$, we may simply use the steady-state limiting forms

$$
C_{\alpha}^{\infty}(t)=R^{-1}\left[(\mathrm{~d} / \mathrm{d} t) \log \left|u_{2}(t)\right|+\lambda\right]-\frac{1}{2} \hat{Q}_{\alpha} \quad(\alpha=\mathrm{A}, \mathrm{B})
$$

and

$$
L^{\infty}(t)=R^{-1}\left\{\left[(\mathrm{~d} / \mathrm{d} t) \log \left|u_{2}(t)\right|+\lambda\right]^{2}-S^{2}\right\}
$$

to describe concentrations and recombination rate.

In addition to these results, averages over a cycle, defined like

$$
\langle F(t)\rangle=\tau_{\mathrm{c}}{ }^{-1} \int_{0}^{\tau_{\mathrm{c}}} F\left(t+t^{\prime}\right) \mathrm{d} t^{\prime},
$$

merit further attention. The average concentrations are readily calculated from (2.6) to give

$$
\left\langle C_{\alpha}(t)\right\rangle=\left(R \tau_{\mathrm{c}}\right)^{-1} \log \left|Z\left(t+\tau_{\mathrm{c}}\right) / Z(t)\right|-\frac{1}{2} \hat{Q}_{\alpha} \quad(\alpha=\mathrm{A}, \mathrm{B})
$$

\footnotetext{
$\dagger$ The restriction to the semi-infinite interval of positive times is irrelevant for the conclusions which follow.
} 
by referring to (2.20) and (2.21) upon recalling (2.10). Similarly, the average recombination rate comes from (2.1) and (2.6):

$$
\langle L(t)\rangle=P_{0}-\frac{1}{R \tau_{\mathrm{c}}} \frac{\mathrm{d}}{\mathrm{d} t} \log \left|\frac{Z\left(t+\tau_{\mathrm{c}}\right)}{Z(t)}\right|
$$

with the constant

$$
P_{0}=\langle P(t)\rangle \text {. }
$$

In the limit of large times, expressions (2.26) and (2.27) yield the steady-state average values

$$
\left\langle C_{\alpha}^{\infty}(t)\right\rangle=\lambda / R-\frac{1}{2} \hat{Q}_{\alpha} \quad(\alpha=\mathrm{A}, \mathrm{B})
$$

and

$$
\left\langle L^{\infty}(t)\right\rangle=P_{0}
$$

to provide the constant components of the fluctuations of both the concentrations and the recombination rate, equations $(2.23)$ and (2.24). This follows immediately by use of the representation $(2.21)$.

\section{Illustrative examples}

Hereafter we give some examples which demonstrate the usefulness of the theoretical framework we have outlined for obtaining analytic solutions of the rate equations with both types of approach, and we shall cover periodic as well as aperiodic timedependences of the production term. For brevity, only the relevant steps and final results will be quoted.

We start with the first method of solution, in which we specify a production rate $P(t)$ and derive the appropriate concentrations and the recombination rate.

(i) Production rate as periodic rectangular pulses

Consider $P(t)$ with the repeatedly stepped form $(n=0,1,2, \ldots)$

$$
P(t)= \begin{cases}P_{\max } & \text { for } n \tau_{\mathrm{c}} \leqslant t<n \tau_{\mathrm{c}}+\tau_{\mathrm{p}} ; P_{\max }>P_{\min } \geqslant 0, \\ P_{\min } & \text { for } n \tau_{\mathrm{c}}+\tau_{\mathrm{p}} \leqslant t<(n+1) \tau_{\mathrm{c}} ; \tau_{\mathrm{c}}>\tau_{\mathrm{p}}>0 .\end{cases}
$$

The maxima have height $P_{\max }$, and duration $\tau_{\mathrm{p}}$; the minima are of height $P_{\min }$, and the cycle length is $\tau_{\mathrm{c}}$. Let the initial values of the concentrations $\hat{C}_{\alpha} \geqslant 0$ for $\alpha=\mathrm{A}, \mathrm{B}$ (both not zero), so that $S>0$. Here (2.7) with (3.1) constitutes Meissner's equation (Strutt 1967) which, in the degenerate case of infinitely high and short 'on-off' pulses, reduces formally to the Kronig-Penney (1931) model for an electron in a onedimensional (semi-infinite) lattice. Defining rate constants $\mu$ and $\nu$ by

$$
\mu=\left(S^{2}+R P_{\max }\right)^{1 / 2}, \quad \nu=\left(S^{2}+R P_{\min }\right)^{1 / 2},
$$

we seek the characteristic exponent $\lambda$ in the ansatz (2.21) for the particular integrals of equation (2.7) suitable for (3.1). Following the appendix, we find that $\lambda$ is determined by the relation

$$
\cosh \left(\lambda \tau_{\mathrm{c}}\right)=\cosh \left(\mu \tau_{\mathrm{p}}\right) \cosh \left[\nu\left(\tau_{\mathrm{c}}-\tau_{\mathrm{p}}\right)\right]+\frac{\mu^{2}+\nu^{2}}{2 \mu \nu} \sinh \left(\mu \tau_{\mathrm{p}}\right) \sinh \left[\nu\left(\tau_{\mathrm{c}}-\tau_{\mathrm{p}}\right)\right]
$$


The appropriate concentrations are $(n=0,1,2, \ldots)$

$C_{\alpha}(t)=\left\{\begin{array}{ll}\frac{\mu}{R} f_{\max }(t)-\frac{1}{2} \hat{Q}_{\alpha} & \text { for } n \tau_{\mathrm{c}} \leqslant t<n \tau_{\mathrm{c}}+\tau_{\mathrm{p}} \\ \frac{\nu}{R} f_{\min }(t)-\frac{1}{2} \hat{Q}_{\alpha} & \text { for } n \tau_{\mathrm{c}}+\tau_{\mathrm{p}} \leqslant t<(n+1) \tau_{\mathrm{c}}\end{array}(\alpha=\mathrm{A}, \mathrm{B})\right.$,

and the recombination rate is obtained $(n=0,1,2, \ldots)$

$$
L(t)= \begin{cases}R^{-1}\left[\mu^{2} f_{\max }^{2}(t)-S^{2}\right] & \text { for } n \tau_{\mathrm{c}} \leqslant t<n \tau_{\mathrm{c}}+\tau_{\mathrm{p}}, \\ R^{-1}\left[\nu^{2} f_{\min }^{2}(t)-S^{2}\right] & \text { for } n \tau_{\mathrm{c}}+\tau_{\mathrm{p}} \leqslant t<(n+1) \tau_{\mathrm{c}} .\end{cases}
$$

In these equations

$$
\begin{aligned}
& f_{\max }(t)=\left(A^{(n)} \mathrm{e}^{\mu t}+B^{(n)} \mathrm{e}^{-\mu t}\right) /\left(A^{(n)} \mathrm{e}^{\mu t}-B^{(n)} \mathrm{e}^{-\mu t}\right), \\
& f_{\min }(t)=\left(C^{(n)} \mathrm{e}^{\nu t}+D^{(n)} \mathrm{e}^{-\nu t}\right) /\left(C^{(n)} \mathrm{e}^{\nu t}-D^{(n)} \mathrm{e}^{-\nu t}\right),
\end{aligned}
$$

with the various coefficients $A^{(n)}, B^{(n)}, C^{(n)}, D^{(n)}$ depending on $n, \tau_{\mathrm{c}}, \tau_{\mathrm{p}}, \mu, \nu, \lambda$ and $S$. In steady state achieved at large times, $t \gg 1 / \lambda$, or, equivalently, after many cycles, $n \gg 1 / \lambda \tau_{c}$, expressions (3.4) and (3.5) simplify to

$C_{\alpha}^{\infty}(t)=\left\{\begin{array}{ll}\frac{\mu}{R} f_{\max }^{\infty}(t)-\frac{1}{2} \hat{Q}_{\alpha} & \text { for } n \tau_{\mathrm{c}} \leqslant t<n \tau_{\mathrm{c}}+\tau_{\mathrm{p}} \\ \frac{\nu}{R} f_{\min }^{\infty}(t)-\frac{1}{2} \hat{Q}_{\alpha} & \text { for } n \tau_{\mathrm{c}}+\tau_{\mathrm{p}} \leqslant t<(n+1) \tau_{\mathrm{c}}\end{array} \quad(\alpha=\mathrm{A}, \mathrm{B})\right.$,

and

$L^{\infty}(t)= \begin{cases}R^{-1}\left[\mu^{2}\left(f_{\max }^{\infty}(t)\right)^{2}-S^{2}\right] & \text { for } n \tau_{\mathrm{c}} \leqslant t<n \tau_{\mathrm{c}}+\tau_{\mathrm{p}}, \\ R^{-1}\left[\nu^{2}\left(f_{\min }^{\infty}(t)\right)^{2}-S^{2}\right] & \text { for } n \tau_{\mathrm{c}}+\tau_{\mathrm{p}} \leqslant t<(n+1) \tau_{\mathrm{c}},\end{cases}$

where

$f_{\max }^{\infty}(t)=\left(b_{2} \mathrm{e}^{\mu\left(t-n \tau_{\mathrm{c}}\right)}-a_{2} \mathrm{e}^{-\mu\left(t-n \tau_{\mathrm{c}}\right)}\right) /\left(b_{2} \mathrm{e}^{\mu\left(t-n \tau_{\mathrm{c}}\right)}+a_{2} \mathrm{e}^{-\mu\left(t-n \tau_{\mathrm{c}}\right)}\right)$,

$f_{\text {min }}^{\infty}(t)=\left(d_{2} \mathrm{e}^{\nu\left(t-n \tau_{\mathrm{c}}\right)}-c_{2} \mathrm{e}^{-\nu\left(t-n \tau_{\mathrm{c}}\right)}\right) /\left(d_{2} \mathrm{e}^{\nu\left(t-n \tau_{\mathrm{c}}\right)}+c_{2} \mathrm{e}^{-\nu\left(t-n \tau_{\mathrm{c}}\right)}\right)$,

with constants $a_{2}, b_{2}, c_{2}, d_{2}$ depending on $\tau_{\mathrm{c}}, \tau_{\mathrm{p}}, \mu, \nu$ and $\lambda$.

(ii) Continuous production rate

Whilst a constant obviously represents a simple function in its own right, it is instructive to consider it as a special limit of the periodic rectangular pulses of example (i). We let $P_{\max }$ and $P_{\min }$ therefore both take the same value, say the average $P_{0}$, thus

$$
P(t)=P_{0} \quad t \geqslant 0 ; \quad P_{0}>0
$$

and assume again $\hat{C}_{\alpha} \geqslant 0$ for $\alpha=\mathrm{A}, \mathrm{B}$ (both not zero), so that $S>0$. The two rate constants defined in (3.2) are then identical and, from (3.3), equal the characteristic exponent which now simply reads

$$
\lambda=\left(S^{2}+R P_{0}\right)^{1 / 2} \text {. }
$$

Hence, the concentrations (3.4) become

$$
C_{\alpha}(t)=(\lambda / R) f(t)-\frac{1}{2} \hat{Q}_{\alpha} \quad \text { for } t \geqslant 0 \quad(\alpha=\mathrm{A}, \mathrm{B})
$$


and the recombination rate (3.5) becomes

$$
L(t)=R^{-1}\left[\lambda^{2} f^{2}(t)-S^{2}\right] \quad \text { for } t \geqslant 0,
$$

where

$$
f(t)=\left(A \mathrm{e}^{\lambda t}+B \mathrm{e}^{-\lambda t}\right) /\left(A \mathrm{e}^{\lambda t}-B \mathrm{e}^{-\lambda t}\right)
$$

with constants given by

$$
A=S+\lambda, \quad B=S-\lambda .
$$

From (3.12) and (3.13) the steady-state limits

$$
C_{\alpha}^{\infty}=\lambda / R-\frac{1}{2} \hat{Q}_{\alpha} \quad(\alpha=\mathrm{A}, \mathrm{B})
$$

and

$$
L^{\infty}=P_{0}
$$

are easily verified in agreement with the previous example.

(iii) Production rate as a single rectangular pulse

This situation too can be considered as a special case of example (i), where the minimum production rate has $P_{\min }=0$ and is indefinitely prolonged. We shall proceed directly, however, and assume

$$
P(t)= \begin{cases}P_{\max } & \text { for } 0 \leqslant t<\tau_{\mathrm{p}} ; P_{\max }>0, \\ 0 & \text { for } \tau_{\mathrm{p}} \leqslant t<\infty ; \tau_{\mathrm{p}}>0 ;\end{cases}
$$

again $\hat{C}_{\alpha} \geqslant 0$ for $\alpha=\mathrm{A}, \mathrm{B}$ (both not zero), so that $S>0$. Equation (2.7) is easily solved with (3.18) for the two time intervals. Following $\S 2.1$, and matching the logarithmic derivative of the appropriate solution $Z(t)$ in the first interval at $t=\tau_{\mathrm{p}}$ smoothly to the respective solution in the second interval, we find, with the definition (3.2), that the concentrations have the form

$$
C_{\alpha}(t)=\left\{\begin{array}{ll}
\frac{\mu}{R} f_{\max }(t)-\frac{1}{2} \hat{Q}_{\alpha} & \text { for } 0 \leqslant t<\tau_{\mathrm{p}} \\
\frac{\nu}{R} f_{\min }(t)-\frac{1}{2} \hat{Q}_{\alpha} & \text { for } \tau_{\mathrm{p}} \leqslant t<\infty
\end{array} \quad(\alpha=\mathrm{A}, \mathrm{B})\right.
$$

extending the result of Capellos and Bielski (1972) which was confined to the case $S=0$. The recombination rate is given by

$$
L(t)= \begin{cases}R^{-1}\left[\mu^{2} f_{\max }^{2}(t)-S^{2}\right] & \text { for } 0 \leqslant t<\tau_{\mathrm{p}}, \\ R^{-1}\left[\nu^{2} f_{\min }^{2}(t)-S^{2}\right] & \text { for } \tau_{\mathrm{p}} \leqslant t<\infty .\end{cases}
$$

The forms of the auxiliary functions are as in (3.6), but now the actual coefficients have the values

$$
\begin{aligned}
& A=S+\mu, \quad B=S-\mu, \\
& C=(S+\mu)^{2} \mathrm{e}^{(\mu-\nu) \tau_{\mathrm{p}}-(S-\mu)^{2} \mathrm{e}^{-(\mu+\nu) \tau_{\mathrm{p}}},} \\
& D=\left(S^{2}-\mu^{2}\right) \mathrm{e}^{-(\mu-\nu) \tau_{\mathrm{p}}-\left(S^{2}-\mu^{2}\right) \mathrm{e}^{(\mu+\nu) \tau_{\mathrm{p}}} .}
\end{aligned}
$$

Expressions (3.19) and (3.20) yield the steady-state limits for the $c$ ncentrations

$$
C_{\alpha}^{\infty}=\hat{C}_{\alpha} \quad(\alpha=\mathrm{A}, \mathrm{B})
$$


and the recombination rate

$$
L^{\infty}=0
$$

as expected.

Figure 1 illustrates forms of the production rate and the relaxation curves derived for the corresponding concentrations and the recombination rate of examples (i), (ii) and (iii) using the specific set of values for the parameters involved defined in the caption.
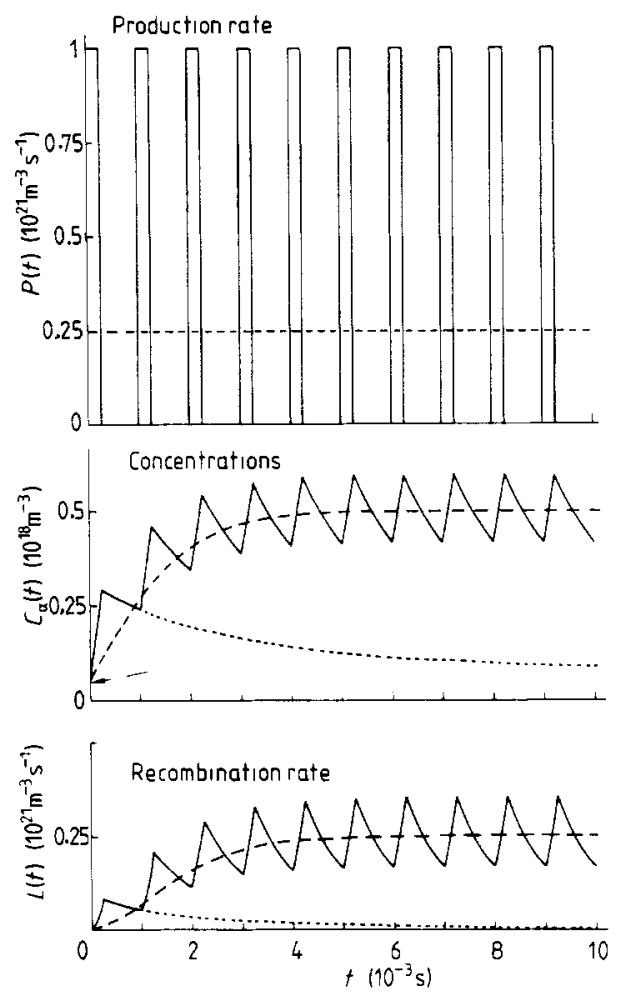

Figure 1. Production rate, concentrations, and recombination rate as functions of time for examples (i) periodic pulses (full lines), (ii) continuous excitation (broken lines), and (iii) a single pulse (dotted lines). Values chosen for the parameters are $P_{\max }=10^{21} \mathrm{~m}^{-3} \mathrm{~s}^{-1}$, $P_{\min }=0, \tau_{\mathrm{c}}=10^{-3} \mathrm{~s}$ and $\tau_{\mathrm{p}}=2.5 \times 10^{-4} \mathrm{~s}$, corresponding to $P_{0}=2.5 \times 10^{20} \mathrm{~m}^{-3} \mathrm{~s}^{-1}$; furthermore $R=10^{-15} \mathrm{~m}^{3} \mathrm{~s}^{-1}$ and $\hat{C}_{\alpha}=5 \times 10^{16} \mathrm{~m}^{-3}$ for $\alpha=\mathrm{A}$, B.

We now turn to the second method of solution, in which we postulate a particular integral $Z_{1}(t)$ of equation (2.7) and deduce the corresponding production rate.

(iv) Exponentially decaying production rate

Suppose $\hat{C}_{\alpha}=0$ for $\alpha=\mathrm{A}$, B, i.e. $S=0$, and try

$$
\begin{array}{lll}
Z_{1}(t)=I_{0}\left(\gamma \mathrm{e}^{-t / 2 \tau}\right) & \text { for } t \geqslant 0 ; & \\
\gamma=\left(4 R P_{\max } \tau^{2}\right)^{1 / 2}, & P_{\max }>0, \quad \tau>0,
\end{array}
$$

with $I_{0}$ a modified Bessel function of the first kind and order zero. From the standard 
recurrence relation this ansatz is seen to satisfy (2.14) and, by reference to (2.13), it generates the function

$$
P(t)=P_{\max } \mathrm{e}^{-t / \tau} \geqslant 0 \quad \text { for } t \geqslant 0
$$

which, being independent of $R$ and bounded, is acceptable for modelling a production rate. With the form $(3.24)$, relation $(2.15)$ clearly allows

$$
Z_{2}(t)=K_{0}\left(\gamma \mathrm{e}^{-t / 2 \tau}\right) \quad \text { for } t \geqslant 0
$$

as a further linearly independent particular integral, where $K_{0}$ denotes a modified Bessel function of the second kind and order zero. Using (3.24) and (3.26) in (2.9) and (2.12), we obtain concentrations

$$
C_{\alpha}(t)=(\gamma / 2 R \tau) g(t) \quad \text { for } t \geqslant 0 \quad(\alpha=\mathrm{A}, \mathrm{B})
$$

and recombination rate

$$
L(t)=\left(\gamma^{2} / 4 R \tau^{2}\right) g^{2}(t) \quad \text { for } t \geqslant 0
$$

with

$$
\begin{gathered}
g(t)=\left\{\left[I_{1}(\gamma) K_{1}\left(\gamma \mathrm{e}^{-t / 2 \tau}\right)-K_{1}(\gamma) I_{1}\left(\gamma \mathrm{e}^{-t / 2 \tau}\right)\right] /\left[I_{1}(\gamma) K_{0}\left(\gamma \mathrm{e}^{-t / 2 \tau}\right)\right.\right. \\
\left.\left.+K_{1}(\gamma) I_{0}\left(\gamma \mathrm{e}^{-t / 2 \tau}\right)\right]\right\} \mathrm{e}^{-t / 2 \tau} .
\end{gathered}
$$

Both vanish in steady state achieved at large times.

(v) Production rate of squared Lorentzian type

Assume again $\hat{C}_{\alpha}=0$ for $\alpha=\mathrm{A}, \mathrm{B}$, i.e. $S=0$, and consider the ansatz

$$
\begin{aligned}
& Z_{1}(t)=\cos \left[\delta \tan ^{-1}(t / \tau)\right]\left(t^{2}+\tau^{2}\right)^{1 / 2} \quad \text { for } t \geqslant 0 ; \\
& \delta=\left(1-R P_{\max } \tau^{2}\right)^{1 / 2}, \quad P_{\max }>0, \quad \tau>0,
\end{aligned}
$$

which satisfies the requirement $(2.14)$, thus ensuring that

$$
P(t)=P_{\max }\left(\frac{\tau^{2}}{t^{2}+\tau^{2}}\right)^{2} \geqslant 0 \quad \text { for } t \geqslant 0
$$

deduced from (2.13) is independent of $R$ and bounded. The form (3.30), as a particular integral of equation (2.7), apparently fulfils (2.16). Thus both the concentrations and the recombination rate are derived immediately using (2.17) and (2.18) to give

$$
C_{\alpha}(t)=R^{-1} h(t) \quad \text { for } t \geqslant 0 \quad(\alpha=\mathrm{A}, \mathrm{B})
$$

and

$$
L(t)=R^{-1} h^{2}(t) \quad \text { for } t \geqslant 0,
$$

where

$$
h(t)=\left\{t-\delta \tau \tan \left[\delta \tan ^{-1}(t / \tau)\right]\right\} /\left(t^{2}+\tau^{2}\right) .
$$

As for the previous example, these quantities vanish in steady state.

Figure 2 illustrates the production rate together with the relaxation curves for the concentrations and the recombination rate of examples (iv) and (v) subject to the particular values for the various parameters defined in the caption. 


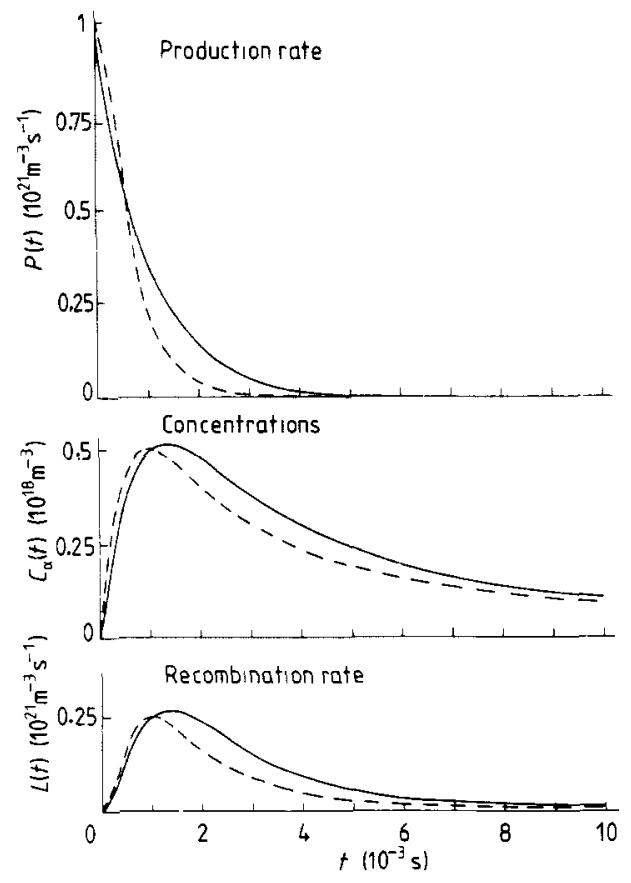

Figure 2. Production rate, concentrations, and recombination rate as functions of time for examples (iv) exponentially decaying production rate (full lines) and ( $v$ ) production rate of squared Lorentzian type (broken lines). Values chosen for the parameters are $P_{\max }=$ $10^{21} \mathrm{~m}^{-3} \mathrm{~s}^{-1}$ and $\tau=10^{-3} \mathrm{~s} ;$ furthermore $R=10^{-15} \mathrm{~m}^{3} \mathrm{~s}^{-1}$ and $\hat{C}_{\alpha}=0$ for $\alpha=\mathrm{A}$, B.

\section{Discussion}

We have studied methods of solving kinetic equations for recombination reactions, in which the production of participating species is driven externally at a time-dependent rate. Although the present analysis refers to reactions among two mobile species, it naturally applies as well to other reactions which can be described by nonlinear rate equations of the Riccati form adopted in $\S 2$. This is true for certain one-species recombination reactions in photoconductivity, e.g. when the holes are entirely trapped and a fraction of the electrons nominally in the conduction band are trapped at any instant, so that electrons alone need to be regarded as mobile reacting species (cf Kittel 1976). The corresponding situation, when both species are mobile, has been considered recently in radiation damage for a constant production rate using perturbation series expansions (Rauh and Simon 1981).

Under some circumstances a theoretical treatment of bimolecular recombination reactions can be facilitated by linearising the basic rate equations with respect to the actual concentrations. However, the full nonlinear analysis becomes indispensable when the concentrations deviate substantially from their initial equilibrium values. This may happen for high species production rates or large times; it is commonly the case for temperatures which are low in relation to the relevant formation energies so that thermal species concentrations are negligible.

The examples we have performed explicitly in $\S 3$ on the basis of the nonlinear rate equations under consideration represent, of course, merely a fraction of the cases 
which are soluble. Other examples include, e.g., a production rate which is sinusoidally modulated about some constant value, or production rates decaying with various inverse power laws in time. The results of such calculations offer a variety of solutions which can be employed to assist identification of the mechanisms operating and to aid extraction of rate constants. This might be done by choosing specific forms of the external field and then comparing the theoretical predictions with the dynamic response of the experimental system manifest in the concentrations and the recombination rate $\dagger$. If, for instance, the experiment is photoexcitation and luminescence of carriers, one would measure the photocurrent and the luminescence intensity as functions of time (Rywkin 1965); in radiation damage different experiments (e.g. measurement of the electrical resistivity) monitor comparable quantities. The fact that analytic solutions are (or can be made) available for production rates with various time-dependences involving parameters subject to external control (e.g. $P_{\max }, P_{\min }, \tau_{\mathrm{c}}$ and $\tau_{\mathrm{p}}$ in the case of example (i) in §3) allows us hereby to try and select the form of the field which leads to an optimal response in the actual physical situation. One immediate advantage, in the light of the formulations of $\$ 2.3$, certainly exists for periodic excitations. Thus estimating the characteristic exponent, i.e. the time required to establish steady state, from the experiment and measuring the constant components (or averages) of the fluctuating concentrations in the ensuing steady-state regime (together with the difference in thermal concentrations) readily provides a lower bound for the recombination constant. Remarkably, this conclusion is true for any periodic excitation and does not necessitate knowing the analytic form of the characteristic exponent at all. Such an approach appears therefore particularly helpful when explicit calculations of the characteristic exponent are difficult.

\section{Acknowledgment}

One of the authors (HR) would like to thank the Commission of the European Communities for the award of a scholarship.

\section{Appendix}

The form (3.1) of the production rate satisfies (2.19) so that the explanations of 82.3 concerning the solution of (2.7) apply. Substituting the ansatz (2.21) yields two equations with constant coefficients for the periodic functions stated in (2.22), which are readily solved to give representations $(n=0,1,2, \ldots)$

$u_{1}(t)= \begin{cases}a_{1} \mathrm{e}^{(\lambda+\mu)\left(t-n \tau_{\mathrm{c}}\right)}+b_{1} \mathrm{e}^{(\lambda-\mu)\left(t-n \tau_{\mathrm{c}}\right)} & \text { for } n \tau_{\mathrm{c}} \leqslant t<n \tau_{\mathrm{c}}+\tau_{\mathrm{p}}, \\ c_{1} \mathrm{e}^{(\lambda+\nu)\left(t-n \tau_{\mathrm{c}}\right)}+d_{1} \mathrm{e}^{(\lambda-\nu)\left(t-n \tau_{\mathrm{c}}\right)} & \text { for } n \tau_{\mathrm{c}}+\tau_{\mathrm{p}} \leqslant t<(n+1) \tau_{\mathrm{c}},\end{cases}$
$u_{2}(t)= \begin{cases}a_{2} \mathrm{e}^{-(\lambda+\mu)\left(t-n \tau_{\mathrm{c}}\right)}+b_{2} \mathrm{e}^{-(\lambda-\mu)\left(t-n \tau_{\mathrm{c}}\right)} & \text { for } n \tau_{\mathrm{c}} \leqslant t<n \tau_{\mathrm{c}}+\tau_{p}, \\ c_{2} \mathrm{e}^{-(\lambda+\nu)\left(t-n \tau_{\mathrm{c}}\right)}+d_{2} \mathrm{e}^{-(\lambda-\nu)\left(t-n \tau_{\mathrm{c}}\right)} & \text { for } n \tau_{\mathrm{c}}+\tau_{\mathrm{p}} \leqslant t<(n+1) \tau_{\mathrm{c}},\end{cases}$

respectively, using the definition (3.2). The pre-exponential constants can be obtained as follows. Since they are solutions of second-order differential equations, both the

$\dagger$ Methods for a systematic analysis of experimental relaxation curves are discussed in detail by Schmid and Sapunov (1982). 
functions $u_{1}(t)$ and $u_{2}(t)$ from (A1) and (A2) and their first derivatives must be continuous at the knot points $t=n \tau_{\mathrm{c}}+\tau_{\mathrm{p}}$. Moreover, the periodic nature of $u_{1}(t)$ and $u_{2}(t)$ imposes the condition that the values of the solutions (A1) and (A2) as well as of their first derivatives at he start of each cycle $t=n \tau_{\mathrm{c}}$ are equal to the respective values at the end of each cycle $t=(n+1) \tau_{\mathrm{c}}$. These requirements entail two sets of linear homogeneous equations for $a_{1}, b_{1}, c_{1}, d_{1}$ and $a_{2}, b_{2}, c_{2}, d_{2}$, which allow non-trivial solutions only if the relation between the characteristic exponent $\lambda$ and the quantities $\mu$ and $\nu$,

$\cosh \left(\lambda \tau_{\mathrm{c}}\right)=\cosh \left(\mu \tau_{\mathrm{p}}\right) \cosh \left[\nu\left(\tau_{\mathrm{c}}-\tau_{\mathrm{p}}\right)\right]+\frac{\mu^{2}+\nu^{2}}{2 \mu \nu} \sinh \left(\mu \tau_{\mathrm{p}}\right) \sinh \left[\nu\left(\tau_{\mathrm{c}}-\tau_{\mathrm{p}}\right)\right]$,

is satisfied. The solutions themselves are determined (up to an arbitrary non-zero factor) to read

$$
\begin{aligned}
& a_{1}=(\lambda-\nu)(\mu+\nu) \mathrm{e}^{\nu\left(\tau_{\mathrm{c}}-\tau_{\mathrm{p}}\right)}-(\lambda+\nu)(\mu-\nu) \mathrm{e}^{-\nu\left(\tau_{\mathrm{c}}-\tau_{\mathrm{p}}\right)}-2 \nu(\lambda-\mu) \mathrm{e}^{\lambda \tau_{\mathrm{c}}-\mu \tau_{\mathrm{p}}}, \\
& b_{1}=-(\lambda+\nu)(\mu+\nu) \mathrm{e}^{-\nu\left(\tau_{\mathrm{c}}-\tau_{\mathrm{p}}\right)}+(\lambda-\nu)(\mu-\nu) \mathrm{e}^{\nu\left(\tau_{\mathrm{c}}-\tau_{\mathrm{p}}\right)}+2 \nu(\lambda+\mu) \mathrm{e}^{\lambda \tau_{\mathrm{c}}+\mu \tau_{\mathrm{p}},} \\
& c_{1}=-(\lambda+\mu)(\mu-\nu) \mathrm{e}^{-\nu \tau_{\mathrm{c}}+\mu \tau_{\mathrm{p}}}-(\lambda-\mu)(\mu+\nu) \mathrm{e}^{-\nu \tau_{\mathrm{c}}-\mu \tau_{\mathrm{p}}}+2 \mu(\lambda-\nu) \mathrm{e}^{-\lambda \tau_{\mathrm{c}}-\nu \tau_{\mathrm{p}}}, \\
& d_{1}=(\lambda+\mu)(\mu+\nu) \mathrm{e}^{\nu \tau_{\mathrm{c}}+\mu \tau_{\mathrm{p}}}+(\lambda-\mu)(\mu-\nu) \mathrm{e}^{\nu \tau_{\mathrm{c}}-\mu \tau_{\mathrm{p}}}-2 \mu(\lambda+\nu) \mathrm{e}^{-\lambda \tau_{\mathrm{c}}-\nu \tau_{\mathrm{p}}},
\end{aligned}
$$

and

$$
\begin{aligned}
& a_{2}=(\lambda-\nu)(\mu+\nu) \mathrm{e}^{-\nu\left(\tau_{\mathrm{c}}-\tau_{\mathrm{p}}\right)}-(\lambda+\nu)(\mu-\nu) \mathrm{e}^{\nu\left(\tau_{\mathrm{c}}-\tau_{\mathrm{p}}\right)}-2 \nu(\lambda-\mu) \mathrm{e}^{-\lambda \tau_{\mathrm{c}}+\mu \tau_{\mathrm{p}}}, \\
& b_{2}=-(\lambda+\nu)(\mu+\nu) \mathrm{e}^{\nu\left(\tau_{\mathrm{c}}-\tau_{\mathrm{p}}\right)}+(\lambda-\nu)(\mu-\nu) \mathrm{e}^{-\nu\left(\tau_{\mathrm{c}}-\tau_{\mathrm{p}}\right)}+2 \nu(\lambda+\mu) \mathrm{e}^{-\lambda \tau_{\mathrm{c}}-\mu \tau_{\mathrm{p}}}, \\
& c_{2}=-(\lambda+\mu)(\mu-\nu) \mathrm{e}^{\nu \tau_{\mathrm{c}}-\mu \tau_{\mathrm{p}}}-(\lambda-\mu)(\mu+\nu) \mathrm{e}^{\nu \tau_{\mathrm{c}}+\mu \tau_{\mathrm{p}}}+2 \mu(\lambda-\nu) \mathrm{e}^{\lambda \tau_{\mathrm{c}}+\nu \tau_{\mathrm{p}}} \\
& d_{2}=(\lambda+\mu)(\mu+\nu) \mathrm{e}^{-\nu \tau_{\mathrm{c}}-\mu \tau_{\mathrm{p}}}+(\lambda-\mu)(\mu-\nu) \mathrm{e}^{-\nu \tau_{\mathrm{c}}+\mu \tau_{\mathrm{p}}}-2 \mu(\lambda+\nu) \mathrm{e}^{\lambda \tau_{\mathrm{c}}-\nu \tau_{\mathrm{p}}}
\end{aligned}
$$

Recalling (2.21) and making use of (A1) and (A2), the required particular integrals of equation (2.7) can be put as $(n=0,1,2, \ldots)$

$Z_{1}(t)= \begin{cases}a_{1} \mathrm{e}^{-(\lambda+\mu) n \tau_{c}} \mathrm{e}^{\mu t}+b_{1} \mathrm{e}^{-(\lambda-\mu) n \tau_{c}} \mathrm{e}^{-\mu t} & \text { for } n \tau_{\mathrm{c}} \leqslant t<n \tau_{\mathrm{c}}+\tau_{\mathrm{p}}, \\ c_{1} \mathrm{e}^{-(\lambda+\nu) n \tau_{\mathrm{c}}} \mathrm{e}^{\nu t}+d_{1} \mathrm{e}^{-(\lambda-\nu) n \tau_{\mathrm{c}}} \mathrm{e}^{-\nu t} & \text { for } n \tau_{\mathrm{c}}+\tau_{\mathrm{p}} \leqslant t<(n+1) \tau_{\mathrm{c}},\end{cases}$

and

$$
Z_{2}(t)= \begin{cases}a_{2} \mathrm{e}^{(\lambda+\mu) n \tau_{\mathrm{c}}} \mathrm{e}^{-\mu t}+b_{2} \mathrm{e}^{(\lambda-\mu) n \tau_{\mathrm{c}}} \mathrm{e}^{\mu t} & \text { for } n \tau_{\mathrm{c}} \leqslant t<n \tau_{\mathrm{c}}+\tau_{\mathrm{p}}, \\ c_{2} \mathrm{e}^{(\lambda+\nu) n \tau_{\mathrm{c}}} \mathrm{e}^{-\nu t}+d_{2} \mathrm{e}^{(\lambda-\nu) n \tau_{\mathrm{c}}} \mathrm{e}^{\nu t} & \text { for } n \tau_{\mathrm{c}}+\tau_{\mathrm{p}} \leqslant t<(n+1) \tau_{\mathrm{c}},\end{cases}
$$

in terms of the characteristic exponent and constants given by equations (A3)-(A5), respectively. The forms (A6) and (A7) lead to concentrations $(n=0,1,2, \ldots)$

$C_{\alpha}(t)=\left\{\begin{array}{ll}\frac{\mu}{R} f_{\max }(t)-\frac{1}{2} \hat{Q}_{\alpha} & \text { for } n \tau_{\mathrm{c}} \leqslant t<n \tau_{\mathrm{c}}+\tau_{\mathrm{p}} \\ \frac{\nu}{R} f_{\min }(t)-\frac{1}{2} \hat{Q}_{\alpha} & \text { for } n \tau_{\mathrm{c}}+\tau_{\mathrm{p}} \leqslant t<(n+1) \tau_{\mathrm{c}}\end{array} \quad(\alpha=\mathrm{A}, \mathrm{B})\right.$,

and to the recombination rate $(n=0,1,2, \ldots)$

$$
L(t)= \begin{cases}R^{-1}\left[\mu^{2} f_{\max }^{2}(t)-S^{2}\right] & \text { for } n \tau_{\mathrm{c}} \leqslant t<n \tau_{\mathrm{c}}+\tau_{\mathrm{p}}, \\ R^{-1}\left[\nu^{2} f_{\min }^{2}(t)-S^{2}\right] & \text { for } n \tau_{\mathrm{c}}+\tau_{\mathrm{p}} \leqslant t<(n+1) \tau_{\mathrm{c}}\end{cases}
$$


In these expressions the auxiliary functions are given by

$$
\begin{aligned}
& f_{\max }(t)=\left(A^{(n)} \mathrm{e}^{\mu t}+B^{(n)} \mathrm{e}^{-\mu t}\right) /\left(A^{(n)} \mathrm{e}^{\mu t}-B^{(n)} \mathrm{e}^{-\mu t}\right), \\
& f_{\min }(t)=\left(C^{(n)} \mathrm{e}^{\nu t}+D^{(n)} \mathrm{e}^{-\nu t}\right) /\left(C^{(n)} \mathrm{e}^{\nu t}-D^{(n)} \mathrm{e}^{-\nu t}\right),
\end{aligned}
$$

where

$$
\begin{aligned}
& A^{(n)}=k_{1} b_{2} \mathrm{e}^{(\lambda-\mu) n \pi_{c}}-k_{2} a_{1} \mathrm{e}^{-(\lambda+\mu) n \tau_{c}}, \\
& B^{(n)}=k_{2} b_{1} \mathrm{e}^{-(\lambda-\mu) n \tau_{c}}-k_{1} a_{2} \mathrm{e}^{(\lambda+\mu) n \tau_{c}}, \\
& C^{(n)}=k_{1} d_{2} \mathrm{e}^{(\lambda-\nu) n \tau_{c}}-k_{2} c_{1} \mathrm{e}^{-(\lambda+\nu) n \tau_{c}}, \\
& D^{(n)}=k_{2} d_{1} \mathrm{e}^{-(\lambda-\nu) n \tau_{c}}-k_{1} c_{2} \mathrm{e}^{(\lambda+\nu) n \tau_{c}},
\end{aligned}
$$

with

$$
k_{1}=S\left(a_{1}+b_{1}\right)-\mu\left(a_{1}-b_{1}\right), \quad k_{2}=S\left(a_{2}+b_{2}\right)+\mu\left(a_{2}-b_{2}\right) .
$$

The limiting forms for the concentrations and the recombination rate in the case $t \gg 1 / \lambda$, i.e. $n \gg 1 / \lambda \tau_{c}$, read:

$$
C_{\alpha}^{\infty}(t)=\left\{\begin{array}{ll}
\frac{\mu}{R} f_{\max }^{\infty}(t)-\frac{1}{2} \hat{Q}_{\alpha} & \text { for } n \tau_{\mathrm{c}} \leqslant t<n \tau_{\mathrm{c}}+\tau_{\mathrm{p}} \\
\frac{\nu}{R} f_{\min }^{\infty}(t)-\frac{1}{2} \hat{Q}_{\alpha} & \text { for } n \tau_{\mathrm{c}}+\tau_{\mathrm{p}} \leqslant t<(n+1) \tau_{\mathrm{c}}
\end{array} \quad(\alpha=\mathrm{A}, \mathrm{B}),\right.
$$

and

$$
L^{\infty}(t)= \begin{cases}R^{-1}\left[\mu^{2}\left(f_{\max }^{\infty}(t)\right)^{2}-S^{2}\right] & \text { for } n \tau_{\mathrm{c}} \leqslant t<n \tau_{\mathrm{c}}+\tau_{\mathrm{p}} \\ R^{-1}\left[\nu^{2}\left(f_{\min }^{\infty}(t)\right)^{2}-S^{2}\right] & \text { for } n \tau_{\mathrm{c}}+\tau_{\mathrm{p}} \leqslant t<(n+1) \tau_{\mathrm{c}},\end{cases}
$$

where

$f_{\max }^{\infty}(t)=\left(b_{2} \mathrm{e}^{\mu\left(t-n \tau_{\mathrm{c}}\right)}-a_{2} \mathrm{e}^{-\mu\left(t-n \tau_{\mathrm{c}}\right)}\right) /\left(b_{2} \mathrm{e}^{\mu\left(t-n \tau_{\mathrm{c}}\right)}+a_{2} \mathrm{e}^{-\mu\left(t-n \tau_{\mathrm{c}}\right)}\right)$,

$f_{\min }^{\infty}(t)=\left(d_{2} \mathrm{e}^{\nu\left(t-n \tau_{\mathrm{c}}\right)}-c_{2} \mathrm{e}^{-\nu\left(t-n \tau_{\mathrm{c}}\right)}\right) /\left(d_{2} \mathrm{e}^{\nu\left(t-n \tau_{\mathrm{c}}\right)}+c_{2} \mathrm{e}^{-\nu\left(t-n \tau_{\mathrm{c}}\right)}\right)$.

This follows from (A8) and (A9) or, more directly, from (2.23) and (2.24) in conjunction with (A2).

The representations (A6) and (A7) also admit the calculation of average concentrations $(n=0,1,2, \ldots)$

$$
\left\langle C_{\alpha}(t)\right\rangle=\left\{\begin{array}{ll}
\left(R \tau_{\mathrm{c}}\right)^{-1} \log \left|g_{\max }(t)\right|-\frac{1}{2} \hat{Q}_{\alpha} & \text { for } n \tau_{\mathrm{c}} \leqslant t<n \tau_{\mathrm{c}}+\tau_{\mathrm{p}} \\
\left(R \tau_{\mathrm{c}}\right)^{-1} \log \left|g_{\min }(t)\right|-\frac{1}{2} \hat{Q}_{\alpha} & \text { for } n \tau_{\mathrm{c}}+\tau_{\mathrm{p}} \leqslant t<(n+1) \tau_{\mathrm{c}}
\end{array} \quad(\alpha=\mathrm{A}, \mathrm{B}),\right.
$$

and the average recombination rate $(n=0,1,2, \ldots)$

$$
\langle L(t)\rangle= \begin{cases}P_{0}-\left(R \tau_{\mathrm{c}}\right)^{-1}(\mathrm{~d} / \mathrm{d} t) \log \left|g_{\max }(t)\right| & \text { for } n \tau_{\mathrm{c}} \leqslant t<n \tau_{\mathrm{c}}+\tau_{\mathrm{p}}, \\ P_{0}-\left(R \tau_{\mathrm{c}}\right)^{-1}(\mathrm{~d} / \mathrm{d} t) \log \left|g_{\min }(t)\right| & \text { for } n \tau_{\mathrm{c}}+\tau_{\mathrm{p}} \leqslant t<(n+1) \tau_{\mathrm{c}} .\end{cases}
$$

Here the auxiliary functions read

$$
\begin{aligned}
& g_{\max }(t)=\left(A^{(n+1)} \mathrm{e}^{\mu\left(t+\tau_{c}\right)}-B^{(n+1)} \mathrm{e}^{-\mu\left(t+\tau_{c}\right)}\right) /\left(A^{(n)} \mathrm{e}^{\mu t}-B^{(n)} \mathrm{e}^{-\mu t}\right), \\
& g_{\min }(t)=\left(C^{(n+1)} \mathrm{e}^{\nu\left(t+\tau_{c}\right)}-D^{(n+1)} \mathrm{e}^{-\nu\left(t+\tau_{c}\right)}\right) /\left(C^{(n)} \mathrm{e}^{\nu t}-D^{(n)} \mathrm{e}^{-\nu t}\right),
\end{aligned}
$$

and the average production rate is given by

$$
P_{0}=P_{\max }\left(\tau_{\mathrm{p}} / \tau_{\mathrm{c}}\right)+P_{\min }\left(\tau_{\mathrm{c}}-\tau_{\mathrm{p}}\right) / \tau_{\mathrm{c}}
$$


From (A16) and (A17) the steady-state limits (2.29) and (2.30) are verified with the aid of (A11).

It should be noted that a problem in radiation damage of metals which is related to our example (i) has been analysed by Dienes (1978) and Krishan (1980), however without considering Floquet's theorem and its consequences and advantages for the solution of the underlying rate equations.

\section{References}

Arfken G 1970 Mathematical Methods for Physicists (New York: Academic) p 403

Capellos C and Bielski B H J 1972 Kinetic Systems. Mathematical Description of Chemical Kinetics in Solution (New York: Wiley) p 110

Curie D 1963 Luminescence in Crystals (New York: Methuen) p 147

Dienes G J 1978 Rad. Effects 36101

Kehlen H, Kuschel F and Sackmann H 1974 Grundlagen der chemischen Kinetik (Braunschweig: Vieweg) Kittel C 1976 Einführung in die Festkörperphysik (München: Oldenbourg) p 727

Krishan K 1980 Rad. Effects 45169

Kronig R de L and Penney W G 1931 Proc. R. Soc. A 130499

Madelung O 1981 Introduction to Solid State Theory (Berlin: Springer) p 409

Meixner J 1956 in Handbuch der Physik ed S Flügge, vol I (Berlin: Springer) p 208

Mort J and Pai D M (eds) 1976 Photoconductivity and Related Phenomena (Amsterdam: Elsevier) p 27

Rauh H and Simon D 1981 Phys. Lett. 81A 285

Rywkin S M 1965 Photoelektrische Erscheinungen in Halbleitern (Berlin: Akademie) p 37

Schmid R and Sapunov V N 1982 Non-Formal Kinetics in Search for Chemical Reaction Pathways (Weinheim: Chemie) p 61

Stoneham A M 1975 Theory of Defects in Solids (Oxford: OUP) p 547

Strutt M J O 1967 Lamésche, Mathieusche und verwandte Funktionen in Physik und Technik (New York: Chelsea) p 39 